\title{
Multi-Feature Hybrid Gait Recognition Algorithm Based on Iterative Closest Point Algorithm
}

\author{
Qiping $\operatorname{Jin}^{12}$ \\ Automation Research Center, Dalian Maritime University \\ Dalian 116026, China \\ E-mail: huying@dlmu.edu.cn
}

\section{Ying Hu}

Automation Research Center, Dalian Maritime University

Dalian 116026, China

\section{Jin Huang}

Automation Research Center, Dalian Maritime University

Dalian 116026, China

\section{Songting Qin}

Automation Research Center, Dalian Maritime University

Dalian 116026, China

\begin{abstract}
Gait, as a typical feature of biology, highlights such advantages as non-contact, non-invasive, difficulty of hiding and disguise etc. so that the gait recognition has become one of the hottest research directions in the biological feature recognition. In this paper, a multi-feature hybrid recognition algorithm is proposed based on the ICP algorithm for a specific gait database. Firstly, the feature point sets of the gait database are classified and the feature curves are generated; then, the ICP matching for each feature curve is implemented in a step by step manner. Finally, an appropriate threshold is selected according to the ROC curve. The experimental results show that the algorithm still has good recognition effect in the condition of low resolution.
\end{abstract}

CENet2015

12-13 September 2015

Shanghai, China

${ }^{1}$ Speaker

${ }^{2}$ This work is supported by the China Fundamental Research Funds for the Central Universities with the No: 3132013334. 


\section{Introduction}

Gait is generally defined as the posture of people walking. In recent years, the gait recognition has been attracted more and more attentions as a research direction in the area of computer vision and biological characteristic. Compared with other biometric technologies (such as fingerprints, iris and face etc.), the gait recognition technology has some features such as contact-free, non-invasive, difficulty in hide and disguise while it is presenting huge advantages in the field of criminal investigation, accession to control system and security monitoring [1].

Recently, many international and domestic research institutes, scholars of university have done a lot of work in the field of gait recognition. Kinds of related matching algorithms and processing methods were proposed. The existing methods for human gait recognition can be divided roughly into two categories: the appearance-based and the model-based approaches. In respect of the model-based approaches, the human body structure is characterized by using the model parameters fitted based on the extracted features. The parameters can be dynamic (e.g., the stride length and speed) or the static body parameters (e.g., the size ratios of various body parts) [2-5]. Compared with the model-based methods, the appearance-based approaches, which employ a compact representation to characterize the motion patterns of the human body, have demonstrated better performance in terms of common databases. In the appearance-based approaches, different types of features (e.g., the whole silhouettes, silhouette width vectors, or Fourier descriptors) are first extracted [6-10]; however, under the appearance-based approaches, other relevant researches mainly recognize the feature by extracting the feature information of human legs and the object cannot be accurately identified because of less and incomplete characteristic information by above methods.

We aim at proposing a multi-feature hybrid matching algorithm of gait recognition based on the appearance approaches. Such algorithm is designed to recognize the motion information of major joints on the human leg and arm. The process is divided two steps: the first is to achieve macroscopical match and then a detail match. Finally, satisfying experimental results can be achieved. It shows that the algorithm still has sound recognition effect under the condition of low resolution.

\section{Gait Database}

There is no uniform international standard database currently available to the majority of researchers. The most common choice is the UCSD database of University of California and CMU database of Carnegie Mellon University, which has a large storage space, long identified time and more other problems; however, the gait recognition technology in our study applies to the video monitoring field and the database it needs can meet different perspectives, different environments and more requirements. In this sense, the database used in this paper is a standard sample database for the specific application of gait comparison on criminal suspects, which is specifically acquired and established by 213 Research Institute of the Ministry of Public Security. It is obtained in a natural manner by using camera for three hundreds and thirty-eight natural persons that wear reflective markers under the condition of analog video monitoring field in a restricted area. The image of different views in database is respectively shown in Fig.1.

The data stored in the database is two-dimensional coordinate points which are formed based on the time sequence by movement of 11 markers installed on each person. These coordinate points are distributed in the head, shoulder, elbow, wrist, waist, knees and feet, which can adequately reflect the gait information of a walking human. The database includes three hundreds and thirty-eight personal two-dimensional coordinate points walking back and forth in 
two time series. It almost contains information of the feature points in all kinds of situations; however, it is hard to recognize the gauge point because of lower image resolution $(640 \times 480)$.

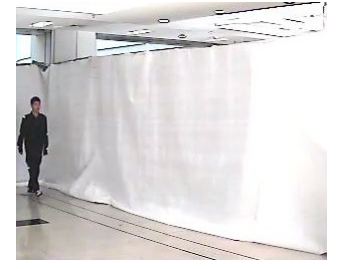

(a) $30^{\circ}$ view

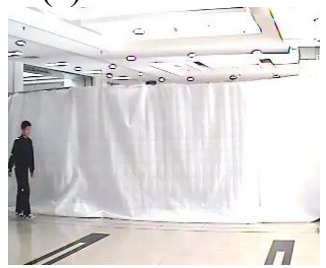

(a) $70^{\circ}$ view

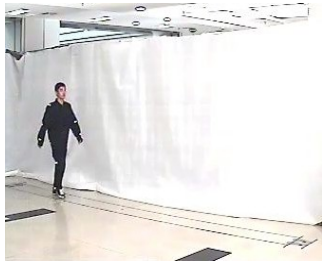

(a) $40^{\circ}$ view

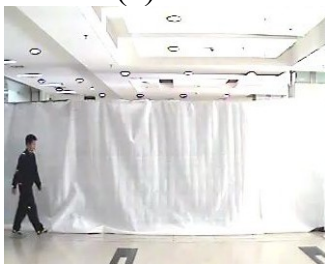

(a) $90^{\circ}$ view

Figure 1: Different View Images

\section{Gait Recognition Method}

The entire gait recognition method is composed of three main parts:1. the moving target detection; 2. the feature extraction; 3. the classification and recognition. Firstly, in order to obtain better binary silhouette image sequence of the walking people, the moving target image sequence that is captured by video surveillance technology needs to be preprocessed and analyzed. Then the feature extraction algorithm is used to extract the gait characteristics information. Finally, by comparing the gait characteristics with those identified with original sample of gait database, the nearest distance classifier is used to identify the staff.

\subsection{ICP Feature Points Matching Algorithm}

ICP, Iterative Closest Point, is an algorithm employed to minimize the difference between two clouds of points. ICP is often used to reconstruct 2D or 3D surfaces from different scans to localize the robots and achieve the optimal path planning (especially when wheel odometry is unreliable due to slippery terrain), co-register the bone models, etc. Since the sample database subject has eleven feature points, there have eleven groups of coordinate point sets. The feature point set of prototypical image is marked as $S$, and $T$ denotes the feature point set of contrast image.

Then the $i$-th prototypical image feature point sets can be written as

$$
S_{i}=\{(x, y) \mid x \in S, y \in S\}
$$

Similarly, the $i$-th contrast image feature point sets can be defined as

$$
T_{i}=\{(x, y) \mid x \in T, y \in T\}
$$

The corresponding point sets registration algorithm in ICP is used to find the coordinate transformation matrix which is approached by the least square [11].

The rotation transformation vector is denoted as unit quaternion $q_{R}=\left[\begin{array}{llll}q_{0} & q_{1} & q_{2} & q_{3}\end{array}\right]^{T}$, where $\mathrm{q}_{0} \geq 0$ and $q_{0}{ }^{2}+q_{1}{ }^{2}+q_{2}{ }^{2}+q_{3}{ }^{2}=1$. Then a $3 \times 3$ rotation matrix $R\left(q_{R}\right)$ can be achieved. 
Suppose the translation transformation vector be expressed as $q_{M}=\left[\begin{array}{lll}q_{4} & q_{5} & q_{6}\end{array}\right]^{T}$, then the coordinate transformation vector is denoted as $q=\left[q_{R} \mid q_{M}\right]^{T}$. It can be obtained fully from above definition; in this sense, the problem of seeking optimum coordinate transformation vector among the corresponding point sets can be transformed into solving $q$ to minimize the function

$$
f(q)=\frac{1}{N_{T}} \sum_{i=1}^{N_{T}}\left\|S_{i}-R\left(q_{R}\right) t_{i}-q_{M}\right\|
$$

Actual matching ratio of the $i$-th feature point is $(1-f(\mathrm{q})) \times 100 \%$

The algorithmic process of the program is shown as follows:

1) Obtain the $i$-th feature point set, ie., $S_{i}$ and $T_{i}$.

2) Calculate the center of gravity of $T_{i}$ and corresponding $S_{i}$ :

$$
\mu_{T_{i}}=\frac{1}{N_{T}} \sum_{i=1}^{N_{T}} t_{i} \quad \mu_{S_{i}}=\frac{1}{N_{S}} \sum_{i=1}^{N_{S}} s_{i}
$$

3) Construct covariance matrix by $T_{i}$ and $S_{i}$ :

$$
\sum T_{i}, S_{i}=\frac{1}{N_{T}} \sum_{i=1}^{N_{T}}\left[\left(t_{i}-\mu_{T_{i}}\right)\left(s_{i}-\mu_{S_{i}}\right)\right]
$$

4) Construct $4 \times 4$ symmetric matrix by covariance matrix:

$$
Q\left(\sum T_{i}, S_{i}\right)=\left[\begin{array}{cc}
\operatorname{tr}\left(\sum T_{i}, S_{i}\right) & \Delta^{T} \\
\Delta & \sum T_{i}, S_{i}+\sum T_{i}, S_{i}^{T}-\operatorname{tr}\left(\sum T_{i}, S_{i}\right) I_{3}
\end{array}\right]
$$

where $\triangle=\left[\mathrm{A}_{21} \mathrm{~A}_{31} \mathrm{~A}_{12}\right]^{T}, \quad \mathrm{~A}_{i, j}=\left(\Sigma_{T i, S i}-\Sigma_{T i, S i}^{T}\right)_{i, j}, I_{3}$ is 3-order matrix, and is trace of matrix $\Sigma_{T i, S i}$

5) Calculate the eigenvalues and eigenvectors of $\mathrm{Q}\left(\Sigma_{T i, S i}^{T}\right)$,whose largest eigenvalues corresponding with eigenvetors is the best rotation vector .

6) Calculate the optimal translation vector, whose calculation expression is

$$
q_{M}=\mu_{S_{i}}-R\left(q_{R}\right) \mu_{T_{i}}
$$

where

$$
R\left(q_{R}\right)=\left(\begin{array}{lll}
q_{0}^{2}+q_{1}^{2}-q_{2}^{2}-q_{3}^{2} & 2\left(q_{1} q_{2}-q_{0} q_{3}\right) & 2\left(q_{1} q_{3}+q_{0} q_{2}\right) \\
2\left(q_{1} q_{2}+q_{0} q_{3}\right) & q_{0}^{2}-q_{1}^{2}+q_{2}^{2}-q_{3}^{2} & 2\left(q_{2} q_{3}-q_{0} q_{1}\right) \\
2\left(q_{1} q_{3}-q_{0} q_{2}\right) & 2\left(q_{2} q_{3}+q_{0} q_{1}\right) & q_{0}^{2}-q_{1}^{2}-q_{2}^{2}+q_{3}^{2}
\end{array}\right)
$$

7) Consider the coordinate transformation vector from the above result, the MMSE and actual matching rate can be obtained as follows:

$$
d_{e s}=f(q) \quad f_{m s}=(1-f(q)) \times 100 \%
$$

With ICP algorithm, the matching rate can be calculated between the prototype and the contrast feature points of each sample person in database. Similarly, the similarity degree of different people is also achieved. 


\subsection{Hybrid Feature Matching Algorithm}

In this section, visual $\mathrm{C}++6.0$ was applied to build a simulation platform by using the above ICP algorithm obtain a personal matching figure of prototype and contrast. The matching results are shown in Fig. 2 and Fig. 3.

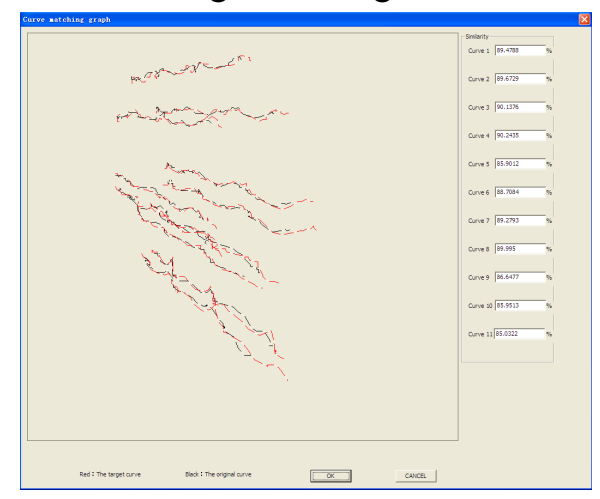

Figure 2: $10^{\circ}$ Prototype and Contrast Matching Chart Figure 3: $60^{\circ}$ Prototype and Contrast Matching Chart

With the accomplishment of abundant matching experiments, the highest fitting degree is presented in the above four curves which correspond to the feature point of the head and upper body in database. While the feature point of arms and legs have relatively low fitting degree.

Thus, the entire matching process is divided into three steps:

1) Firstly, match the height feature of object with sample database library. After that, select the sample person whose height error is less than $10 \mathrm{~cm}$ in this experiment and mark them. Thus, the number of staff in the sample database can be reduced.

2) The stride length of sample person can be calculated according to the coordinate value of feature point in the database. Choose the samples with the error between the step size and the staff selected in step (1) is less than $5 \mathrm{~cm}$.

3) The retaining feature information of leg and upper joint was matched after the above two steps. During the matching process, the sample data which is bigger than the threshold value in the experimental results and has the highest similarity is the best result.

The above-mentioned method avoids seeking the entire staff data of database for each inquiry. Thus, the recognition rate and efficiency of the recognition approaches have been improved effectively.

\section{Experimental Results}

At present, there hasn't been any unified evaluation method in the field of gait recognition. The evaluation index adapted in this experiment referred to the evaluation of fingerprint identification. The efficiency was measured by the FRR and FAR [12]. FRR means the percentage of the rejected number of the matched sample in the total number of test sample. FAR denotes the accepted number of the matched sample in the total number of test sample. According to the research of many scholars, the ROC curve about FRR and FAR is usually drawn by using the cross-validation method, which is defined as that the test subject is extracted in turn from the database to match the rest of the sample. The best threshold can be achieved when FRR is Equal to FAR. 
In order to evaluate our algorithm, 50 groups of feature points are selected from the specific database to match in pairs sequentially. Change the matching rate from $0 \%$ to $100 \%$ and compare the matching rate with the result of Formula (7). Thus ROC curve (as shown in Fig. 4) is achieved. The threshold set as $85 \%$ can be obtained from Fig. 4 .

Using the above threshold, we can draw CMS curve which is cumulative recognition rate. The gait similarity that is greater than $85 \%$ between the person to be recognized and the sample person can be calculated and ranked from large to small; thus we've achieved the CMS curve as shown in Fig.5. When the rank is greater than or equal to 6, the desired recognition rate over 0.9 can be achieved.

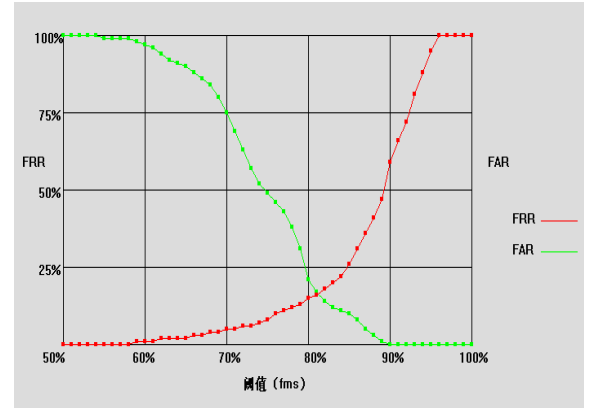

Figure 4: the ROC Curve

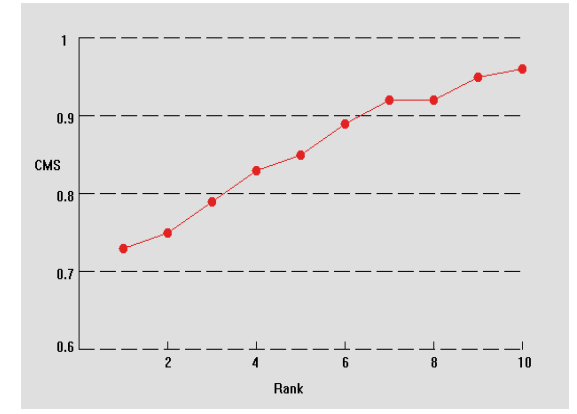

Figure 5: the CMS Curve

\section{Conclusion}

In this paper, a multi-feature hybrid recognition algorithm based on ICP algorithm is designed for specific gait database. The algorithm has reduced the FRR and FAR and improved the system's recognition performance. Experimental results show that the algorithm still has good recognition effect under the condition of low resolution. This algorithm still has some problems yet. For example, it would need considerable long time to run ICP algorithm; therefore, the following problems would be solved successively in the future research. Optimize the ICP algorithm firstly. Design a method to reduce FRR and FAR secondly. Finally, integrate every link of the gait recognition system so that the method may achieves satisfactory effect in practical application.

\section{References}

[1] CK.J. Wang, B.B. Hou. A Survey of Gait Recognition. Journal of Image and Graphics, vol. 12 no. 7, pp. 1152-1160 (2007)(In chinese)

[2] M. Hofmann, J. Geiger, S. Bachmann,B. Schuller,G. Rigoll. The TUM Gait from Audio,Image and Depth(GAID) database:Multimodal recognition of subjects and traits. Journal of Visual Communication and Image Representation, vol. 25 no. 1,pp. 195-206(2014)

[3] F. Zhang, X.H. Zhang, K. Cao, R. Li. Contour extraction of gait recognition based on improved GVF Snake model. Computer \&Electrical Engineering, vol. 38 no. 4, pp. 882-890(2012)

[4] S.Sarkar, P. J. Phillips, Z. Liu, I. R. Vega, P. Grother, K.W. Bowyer. The HumanID gait challenge problem: Data sets, performance, and analysis. IEEE Trans. Pattern Anal. Mach. Intell., vol. 27 no. 2, pp. 162-177 (2005)

[5] E. Zhang, Y.W. Zhao, W. Xiong. Active energy image plus 2DLPP for gait recognition. Signal Processing, vol. 90 no. 7, pp. 2295-2302(2010) 
[6] B. Lee, S.J. Hong, H. Lee. E. Kim. Gait recognition system using decision-lever fusion. IEEE Conference on Industrial Electronics and Applicationsis, Taichung,pp. 313-316(2010)

[7] S. D. Choudhury, T. Tjahjadi. Silhouette-based gait recognition using Procrustes shape analysis and elliptic Fourier descriptors. Iberian Conference on Pattern Recognition and Image Analysis. vol. 45 no. 9 , pp.3414-3426(2012)

[8] P. Das, D. Das, S. Saharia. Gait Analysis and Recognition for Human Identification. International Journal of Electronics and Applied Reseach(IJEAR), pp. 45-54(2014)

[9] Z.Liu, S. Sarkar. Improved gait recognition by gait dynamics normalization. IEEE Trans. Pattern Anal. Mach. Intell., vol. 28, no. 6, pp. 863-876(2006)

[10] S.Yu, D.Tan, T. Tan. Modelling the effect of view angle variation on appearance-based gait recognition. In Proc. Asian Conf. on Computer Vision,vol. 3851 pp. 807-816(2006)

[11] J.L. Dai, Z.Y. Chen, X.Z. Ye. The Application of ICP Algorithm in Point Cloud Alignment. Journal of Image and Graphics, vol. 12 no. 3, pp. 517-521 (2007)

[12] Y.P. Li, Y.X. Wang,X.S. Mou, L. Rong. Study on FRR and FAR test method of fingerprint recognition technology application products . China Public Security. Academy Edition, no. 2, pp. 98-104(2005) 